\title{
Uso de toxina botulínica en neurología: Experiencia en el Hospital Guillermo Almenara Irigoyen, EsSalud
}

\author{
ÉDGARD ROJAS ${ }^{1}$, CÉSAR CASTAÑEDA $^{1}$, ROBERTO PORTILLO $^{2}$, DAVID LIRA $^{3}$ \\ ${ }^{1}$ Médico Neurólogo del Hospital Guillermo Almenara Irigoyen, Servicio de Neurología. Lima, Peru. ${ }^{2}$ Jefe de \\ Servicio de Neurología, Hospital Guillermo Almenara Irigoyen, Lima-Peru. ${ }^{3}$ Residente de Neurología del Servicio. \\ Hospital Guillermo Almenara Irigoyen, Lima-Peru.
}

\begin{abstract}
Resumen
Objetivo: Identificar las principales utilidades de la toxina botulínica en las enfermedades neurológicas. Lugar: Hospital Nacional Guillermo Almenara Irigoyen, EsSalud, Lima,Perú. Periodo 2001-2003. Material y Métodos: Se estudió 122 pacientes a quienes se les infiltró toxina botulínica tipo A a nivel de los músculos comprometidos, según el tipo de patología. Se les hizo una evaluación posterior a la aplicación de la toxina. Resultados: El espasmo hemifacial fue el diagnóstico más frecuente de uso $(63,1 \%)$ seguido de la espasticidad $(16,4 \%)$ y distonía cervical $(11,5 \%)$. El espasmo hemifacial se presentó con más frecuencia entre los 60 y 69 años y en el lado izquierdo. En la distonía cervical, el tipo más frecuente de uso fue en la tortícolis y laterocolis. La evolución fue favorable, según el puntaje de la escala de TWSTRS aplicada antes y 3 meses después del tratamiento. Los efectos secundarios encontrados con más frecuencia fueron el ojo seco (15,6\%), equimosis $(9,1 \%)$ y dolor en el sitio de aplicación $(7,8 \%)$, de naturaleza reversible. Conclusión: La toxina botulínica tipo A tiene múltiples aplicaciones en las enfermedades neurológicas, con buen resultado y pocos efectos secundarios.
\end{abstract}

Palabras clave: Toxinas botulínicas; espasmo; enfermedades neuromusculares.

\section{Botulinum toxin use in neurology: Experience at Guillermo Almenara Irigoyen EsSalud Hospital}

\section{Abstract}

Objective: To identify the utility, characteristics, outcome and adverse effects in the use of botulinum toxin in neurological disorders. Setting: EsSalud's Guillermo Almenara National Hospital, Lima, Peru. Materials and Methods: One hundred and twenty-two patients received botulinum toxin type A in different neurological disorders. We determined the characteristics, outcome and adverse effects with the use of botulinum toxin. Results: The most frequent diagnosis was hemifacial spasm $(63,1 \%)$, followed by spasticity $(16,4 \%)$ and cervical dystonia $(11,5 \%)$. The age of hemifacial spasm most frequent presentation was 60 to 69 year-old and in the left side. Torticollis and laterocollis were the most frequent types of cervical dystonia where botulinum toxin was used. There was good response in the treatment according to the favorable outcome by the TWSTRS scale performed before and 3 months posterior to treatment. The main reversible adverse effects were dry eye $(15,6 \%)$, ecchymosis $(9,1 \%)$ and pain at the site of application $(7,8 \%)$. Conclusion: Botulinum toxin type A has many applications in neurological disorders, with good response and few adverse effects.

Key words: Botulinum toxins; spasm; neuromuscular diseases.

\section{INTRODUCCIÓN}

El uso de la toxina botulínica en el tratamiento de diversas enfermedades se ha ido extendiendo en los últimos años gracias a sus propiedades de bloqueador reversible de la transmisión colinérgica, que lo hacen muy útil en diferentes enfermedades neurológicas.
Desde su utilización por primera vez por Scott para algunas enfermedades neuroftalmológicas $\left({ }^{1}\right)$, en 1970 , hasta nuestros dias, se ha reportado sus múltiples usos en distintas patologías, con relativo éxito. Dentro de las principales enfermedades en las cuales se utiliza la toxina botulínica se encuentran el espasmo 
hemifacial, la distonía cervical, la espasticidad, el blefaroespasmo, la hiperhidrosis palmar y otras distonías más raras, como la distonía de miembros y la distonía oromandibular.

El espasmo hemifacial es un trastorno crónico que se caracteriza por contracciones unilaterales involuntarias, irregulares, clónicas o tónicas de los músculos inervados por el nervio facial; compromete los músculos orbiculares y luego se extiende a los músculos de la parte inferior de la cara. En algunos casos, puede hacerse bilateral. Los movimientos se agravan con la ansiedad, fatiga, lectura, conducción y por movimientos voluntarios de la cara. En algunos casos, con la progresión del cuadro pueden afectarse casi todos los músculos de la cara y músculos superficiales del cuello ( $\left.{ }^{2}\right)$. Los músculos que más se comprometen son el orbicular de los ojos, frontalis, cigomático mayor y menor, orbicular de los labios y borla del mentón. Si bien es cierto, la causa por la cual se produce el espasmo hemifacial no está bien dilucidada, en la mayoría de casos se sabe que la irritación del nervio facial por vasos sanguíneos tortuosos aberrantes que lo comprimen en la fosa posterior después de su salida del tronco cerebral, producen un área focal de desmielinización que genera impulsos ectópicos de transmisión efáctica del impulso a lo largo del nervio facial, que es el que genera los movimientos sincrónicos de la cara observados en el examen físico $\left({ }^{2}\right)$. Otras causas que puedan explicar su fisiopatología incluyen: estimulación aberrante de los núcleos del nervio facial, presencia de aneurismas, malformaciones arteriovenosas, infarto lacunar de la protuberancia, tumores, alteraciones óseas que compriman el nervio, traumatismos, parálisis facial, esclerosis múltiple y hasta causas familiares y psicógenas $\left({ }^{3}\right)$.

El blefaroespasmo es una distonía craneal que se caracteriza por espasmos repetidos y prolongados del músculo orbicular de los párpados $\left({ }^{4}\right)$. La contracción de este músculo origina el cierre palpebral, provocando dificultad para la visión. También es común el parpadeo frecuente, que dificulta las actividades de la vida diaria del paciente.

El tratamiento con toxina botulínica para este tipo de patología y el síndrome de Meige, fue uno de los primeros en mostrar su eficacia en distonías $\left({ }^{5}\right)$. La inyección subcutánea en los extremos de los párpados resultó de mucha utilidad para evitar el cierre ocular y el parpadeo excesivo.

Las causas del blefaroespasmo aún no han sido dilucidadas del todo, permaneciendo en la mayoría de casos como idiopática. Sin embargo, se ha mencionado trastornos genéticos y pérdida neuronal y gliosis en el núcleo caudado y cuerpo estriado, como posibles causas $\left({ }^{6}\right)$.

El síndrome de Meige, caracterizado por blefaroespasmo junto con espasmos de la musculatura facial inferior, considerado como la distonía craneal más frecuente $\left({ }^{3}\right)$, responde adecuadamente al tratamiento con toxina botulínica. Su etiología todavía no está del todo aclarada; sin embargo, existen causas genéticas, debido a exposición por neurolépticos, encefalopatía anóxica, trastornos metabólicos, traumatismos y enfermedades degenerativas $\left({ }^{3}\right)$.

La distonía oromandibular y la distonía de miembros son distonías de rara presentación, correspondiendo a distonías de tipo focal. Son distonías poco frecuentes ( 29,5 por 100,000 habitantes) y responden bien al tratamiento con toxina botulínica. En algunos casos, es necesario ayuda electromiográfica para localizar a los músculos afectados.

La distonía cervical es una distonía focal de torsión que afecta los músculos que controlan la posición de la cabeza $\left(^{7}\right)$. Es más frecuente en adultos y es la segunda indicación en frecuencia de aplicación de toxina botulínica con relativo 
éxito. En nuestro estudio, 14 pacientes, que representaron $11,5 \%$, recibieron dicho tratamiento.

Existen varios tipos de distonía cervical: la forma rotacional o tortícolis propiamente dicha, que es la más común en presentación, el laterocolis, anterocolis, retrocolis y las formas de patrones mixtos. Los músculos más comprometidos son el esternocleidomastoideo, trapecio, esplenius de la cabeza, semiespinal de la cabeza, longissimus de la cabeza, los escalenos y el elevador de la escápula. Las dosis a aplicar varían de acuerdo al tipo de distonía y los músculos involucrados, no sobrepasando la dosis de 300 unidades por sesión, según las recomendaciones internacionales $\left({ }^{7}\right)$.

Varios estudios han mostrado la eficacia de la toxina botulínica en estudios prospectivos doble ciego. El porcentaje de mejoría varía entre 20 y $87 \%$, según diferentes estudios, y generalmente este porcentaje aumenta con la segunda aplicación $\left(^{7-9}\right)$.

La espasticidad es un desorden del sistema sensitivomotor caracterizado por incremento del tono muscular, con reflejos tendinosos exagerados, resultado de la hiperexcitabilidad del reflejo de estiramiento. Es un componente de signo «positivo» del síndrome de primera neurona motora. Se produce por hiperexcitabilidad de la motoneurona alfa al producirse algún tipo de lesión a nivel de la vía piramidal $\left({ }^{10}\right)$. Las lesiones medulares producen espasticidad con una débil excitabilidad y sobreactividad en músculos flexores y extensores, con reacciones que ocurren en muchos segmentos fuera del estímulo. Las lesiones cerebrales, en cambio, producen excitación rápidamente creciente, con un sesgo sobre los músculos antigravitatorios $\left({ }^{11}\right)$.

Estos hallazgos en el paciente con espasticidad, limitan mucho sus actividades de la vida diaria. Afortunadamente, uno de los tratamientos más efectivos en este mal resultó el uso de toxina botulínica. Esto fue corroborado por múltiples estudios clínicos en pacientes con espasticidad por diferentes causas $\left({ }^{11}\right)$.

Las dosis utilizadas en estos casos varían de acuerdo al tamaño y volumen de los músculos involucrados y del objetivo que se quiera lograr con la terapia con toxina botulínica. Es bueno recordar que este tratamiento debe ser necesariamente complementado con terapia física, para lograr mejorar la función motora deseada.

La hiperhidrosis palmar es un fenómeno maladaptativo en el cual se secreta una cantidad de sudor anormalmente superior a la requerida para el balance térmico normal $\left({ }^{12}\right)$. Resulta de una sobreactividad de las glándulas sudoríparas exocrinas que, en este caso, afectan las palmas de las manos y son muy incómodas para que el paciente realice cualquier actividad de tipo manual. En la mayoría de casos, es de causa idiopática.

La infiltración de toxina botulínica tipo A a nivel subcutáneo en diferentes puntos de la palma de la mano, han mejorado mucho a nuestros pacientes en cuanto a la disminución en la sudoración. Si bien los dos casos reportados mostraron una mejoría ostensible, consideramos que sería conveniente evaluar más casos, teniendo en cuenta que la técnica a aplicar requiere mucha destreza y es muy dolorosa, si es que no se utiliza los medios anestésicos adecuados.

A continuación mostramos la experiencia de nuestro hospital en los últimos cuatro años.

\section{MATERIAL Y MÉTODOS}

Se estudió 122 pacientes a quienes se les aplicó toxina botulínica tipo $\mathrm{A}$, en el Servicio de Neurologia del Hospital Guillermo Almenara Irigoyen, de EsSalud. 
Tabla 1. Diagnósticos de aplicación de toxina botulínica en los pacientes del HGAI.

\begin{tabular}{lcr}
\hline Patología & frecuencia & \multicolumn{1}{c}{$\%$} \\
\hline Espasmo hemifacial & 77 & 63,1 \\
Distonía cervical & 14 & 11,5 \\
Blefaroespasmo & 3 & 2,5 \\
Distonía oromandibular & 2 & 1,6 \\
Distonía de miembros & 2 & 1,6 \\
Espasticidad & 20 & 16,4 \\
S.Meige & 2 & 1,6 \\
Hiperhidrosis palmar & 2 & 1,6 \\
Total & 122 & 100,0 \\
\hline
\end{tabular}

En su aplicación se utilizó toxina botulinica tipo A (Botox), que es la única disponible en la actualidad en nuestro medio.

Tabla 2. Numero de aplicaciones de toxina botulínica por paciente.

\begin{tabular}{ccr}
\hline $\mathrm{N}^{\mathbf{0}}$ de aplicaciones & $\mathrm{N}^{\mathbf{0}}$ de casos & \multicolumn{1}{c}{$\%$} \\
\hline 1 & 60 & 49,2 \\
2 & 27 & 22,1 \\
3 & 18 & 14,8 \\
4 & 10 & 8,2 \\
5 & 6 & 4,9 \\
6 & 1 &, 8 \\
Total & 122 & 100,0 \\
\hline
\end{tabular}

Esta toxina, una vez retirada de su cadena de frío, fue preparada con suero fisiológico a diversas concentraciones, según el tipo de músculo aplicado. Las dosis variaron de acuerdo al tipo de

Tabla 3. Número de aplicaciones de toxina botulínica en espasmo hemifacial

\begin{tabular}{ccc}
\hline $\mathrm{N}^{\circ}$ de aplicaciones & $\mathrm{N}^{\circ}$ de casos & $\%$ \\
\hline 1 & 38 & 49,4 \\
2 & 16 & 20,8 \\
3 & 14 & 11,5 \\
4 & 5 & 04,1 \\
5 & 3 & 02,5 \\
6 & 1 & 08,0 \\
Total & 77 & 100,0 \\
\hline
\end{tabular}

Número de aplicaciones promedio por paciente: media $=1,99$ patología aplicada. En algunos casos, fue necesario el apoyo de electromiografía para ubicar músculos profundos a ser infiltrados. El seguimiento de los pacientes fue realizado por un año para ver su evolución.

\section{RESULTADOS}

Como se puede notar en la Tabla 1, la mayor frecuencia de patología en la que se utilizó la toxina botulínica fue el espasmo hemifacial, en más de la mitad de los casos (63\%). Las otras dos patologías de mayor frecuencia en la terapia con toxina botulínica fueron la espasticidad y la distonía cervical, respectivamente (16 y $14 \%)$.

El número de aplicaciones por paciente varió de 1 a 6 en diferentes períodos de tiempo; más de la mitad de los pacientes requirió de la aplicación de toxina botulínica en una sola oportunidad, en tanto que un paciente requirió dicha aplicación en 6 oportunidades durante este lapso (13 meses) (Tabla 2).

El número de aplicaciones para el caso de espasmo hemifacial fue 1 en la mitad de pacientes, el resto requirió aplicaciones periódicas que no sobrepasaron el número de 6. La dosis promedio por paciente fue de 2 (Tabla 3 ).

El espasmo hemifacial fue más frecuente en el sexo femenino, tal como se encuentra en otros trabajos publicados al respecto $\left({ }^{2}\right)$ corres-pondiendo a un $67,5 \%$ de los casos (Tabla 4).

Tabla 4. Sexo de los pacientes con espasmo hemifacial tratados con toxina botulínica.

\begin{tabular}{lcc}
\hline Sexo & $\mathrm{N}^{\circ}$ de casos & $\%$ \\
\hline Masculino & 25 & 32,5 \\
Femenino & 52 & 67,5 \\
\hline Total & 77 & 100,0
\end{tabular}


Tabla 5. Lado comprometido en el espasmo hemifacial.

\begin{tabular}{lcr}
\hline Lado & N $^{\circ}$ de casos & \multicolumn{1}{c}{$\%$} \\
\hline Izquierdo & 44 & 57,1 \\
Derecho & 29 & 37,7 \\
Bilateral & 04 & 05,2 \\
Total & 77 & 100,0 \\
\hline
\end{tabular}

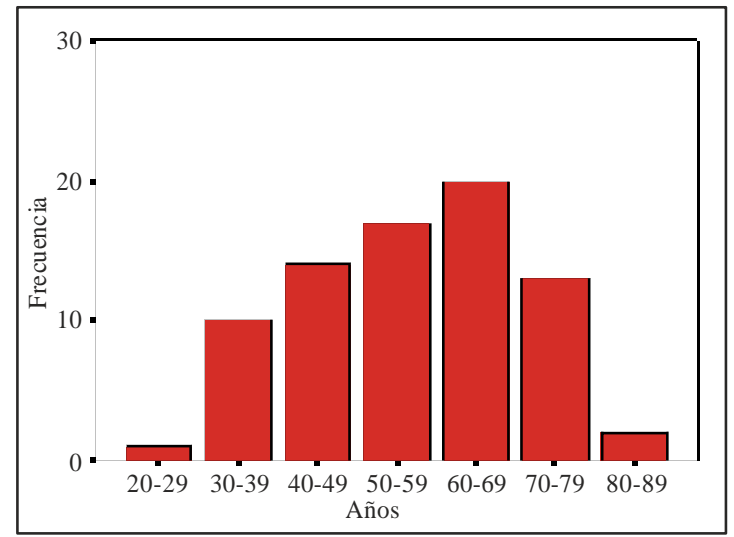

Figura 1. Edad de los pacientes con espasmo hemifacial tratados con toxina botulinica.

Tabla 6. Tipos de distonía cervical.

\begin{tabular}{lrr}
\hline & $\mathrm{n}$ & \multicolumn{1}{c}{$\%$} \\
\hline Tortícolis (rotación) & 7 & 50,0 \\
Laterocolis & 6 & 42,9 \\
Retrocolis & 1 & 7,1 \\
Anterocolis & 0 & 0,0 \\
Total & 14 & 100,0
\end{tabular}

Tabla 7. Evaluación de la distonía cervical con escala de TWSTRS antes y después del tratamiento con toxina botulínica.

\begin{tabular}{lcc}
\hline $\begin{array}{l}\text { Puntaje } \\
\text { TWSTRS }\end{array}$ & $\begin{array}{c}\text { Antes del } \\
\text { tratamiento }\end{array}$ & $\begin{array}{c}\text { Después del } \\
\text { tratamiento }\end{array}$ \\
\hline $0-20$ & 1 & 4 \\
$21-40$ & 4 & 7 \\
$41-60$ & 9 & 3 \\
$61-80$ & 0 & 0 \\
$81-100$ & 0 & 0 \\
Total & 14 & 14 \\
\hline
\end{tabular}

La edad de los pacientes con espasmo hemifacial osciló entre 20 y 89 años. El grupo etario más frecuente fue 60 a 69 años (Figura 1).

El lado más comprometido fue el izquierdo en más de la mitad de los casos $(57,1)$. y el compromiso bilateral el menos frecuente $(5,2 \%)$ (Tabla 5 ).

En el caso de la distonía cervical, el tipo más frecuente de presentación fue la forma de rotación o tortícolis espasmódica, seguida del laterocolis. No tuvimos algún caso de anterocolis. Nuestros pacientes mostraron mejoría parcial en su primera aplicación. Durante su seguimiento, se les evaluó mediante la escala de TWSTRS (Toronto Spasmodic Torticollis Rating Scale) después de 3 meses de tratamiento, mostrando una mejoría en el puntaje. Esta escala evalúa las características del movimiento distónico, la discapacidad que produce el cuadro en las diferentes actividades de la vida diaria y el dolor producido por la distonía $\left(^{8}\right)$ (Tablas 6 y 7 ).

Los efectos secundarios más frecuentes en los casos de blefaroespasmo y espasmo hemifacial fueron el ojo seco y las equimosis, en menos del $18 \%$ de pacientes. Ambos efectos secundarios felizmente de fácil solución, mediante el uso de lágrimas naturales y la apropiada compresión del sitio de inyección que revertían a los pocos días. No tuvimos complicaciones mayores, como ectropión o entropión o presencia de úlceras

Tabla 8. Efectos secundarios al uso de toxina botulínica en blefaroespasmo y espasmo hemifacial.

\begin{tabular}{lrr}
\hline Efectos secundarios & $\mathrm{n}$ & $\%$ \\
\hline Ptosis palpebral & 3 & 3,9 \\
Equimosis & 7 & 9,1 \\
Dolor en sitio de infiltración & 6 & 7,8 \\
Ojo seco & 12 & 15,6 \\
Cefalea & 4 & 5,2 \\
Malestar debilidad & 3 & 3,9 \\
\hline Total & 35 & 45,4
\end{tabular}


corneales (Tabla 8).

En cuanto a la espasticidad, fueron 20 pacientes los que recibieron tratamiento debido a secuela de lesiones medulares, esclerosis múltiple, secuelas de enfermedad vascular cerebral y parálisis cerebral infantil, entre otras causas, las que obtuvieron buena respuesta a la aplicación de toxina botulínica. En la mayoría de casos, este efecto tuvo una duración de cuatro a cinco meses, período en el cual el paciente recibió terapia de rehabilitación, mejorando en la ejecución de algunas actividades de la vida diaria.

Solamente tuvimos dos pacientes con hiperhidrosis palmar, las cuales evolucionaron favorablemente con mejoría ostensible después de la inyección de toxina botulínica. El efecto duró en promedio unos 4 meses. Actualmente, se hallan en seguimiento para aplicaciones posteriores de toxina.

\section{DISCUSIÓN}

Las múltiples aplicaciones que actualmente se dan para el campo de la neurología con la toxina botulínica tipo A, se ven expresados en la experiencia mostrada en nuestro Servicio.

En el espasmo hemifacial, las características demográficas presentadas en cuanto a la edad y predominancia del sexo femenino así como el lado de la lesión comprometido con más frecuencia, coinciden con lo encontrado en otros trabajos $\left({ }^{2}\right)$. El promedio de aplicaciones y el lapso entre aplicación se encuentra dentro del rango permitido para evitar presencia de resistencia por anticuerpos $\left({ }^{13}\right)$.

En el caso de la distonía cervical, la mayor frecuencia de casos fue para el tipo de tortícolis y laterocolis, coincidiendo con lo encontrado en otros trabajos $\left({ }^{9}\right)$.

En las otras patologías, si bien es cierto el número de casos no fue grande, tuvieron una buena eficacia de tratamiento.

Los efectos adversos encontrados no fueron severos y mostraron su reversibilidad. de acuerdo a las características del fármaco. Esto demuestra su gran seguridad para el tratamiento de movimientos involuntarios y espasticidad en nuestros pacientes.

\section{REFERENCIAS BIBLIOGRÁFICAS}

1. Scott AB. Clostridial toxins as therapeutic agents. En: Simpson LL, editor. Botulinum neurotoxins and tetanus toxin. New York: Academic Press; 1989. p. 399-412.

2. Venegas E. Hemiespasmo facial primario y sincinesias postparalíticas tratados con toxina botulínica. Rev Chilena Neuropsiquiatr. 1995;33 Supl 7:S130.

3. López del Val LJ, Castro García A. Toxina botulínica: Aplicaciones terapéuticas. Barcelona: Masson; 2003.

4. Dressler D. Tratamiento con toxina botulinica. México: Masson Doyma; 2003.

5. Nussgens Z. Roggenkamper P. Long term treatment of blepharoespasm with botulinum toxin type A. Ger J Ophtalmol. 1995; 4:363-7.

6. Berardelli A. Rothwell JC, Day BI, Marsden CD. Pathophisiology of blepharoespasm and oromandibular dytonia. Brain .1985;108:593-608.

7. Calne S. Local treatment of dystonia and spasticity with inyections of botulinum-A toxin. Axone. 1993;14:85-8.

8. Jankovic J, Hallett M. Therapy with botulinum toxin. Marcel Decker Inc; 2000.

9. Kessler KR, Skutta M, Benecke R. Long term treatment of cervical dystonia with botulinum toxin A. Efficacy,safety and antibody frequency. German Dystonia Study Group. J Neurol. 1999;246(4):265-74.

10. Gracies J, Elovic E, Mc Guire JM, Simpson DM. Tradicional pharmacological treatments for spasticity. Etiology, evaluation, management and role of Botulinum toxin. New York: We move; 2002.

11. Young R. Spasticity: a review. Neurology. 1994;44 Supl 9:S512-20.

12. Naver H, Aquilonius SM. The trreatment of focal hyperhidrosis with botulinum toxin. Eur J Neurol. 1997;(2):75-9.

13. Tsui JK. Wong E, Calne DB. Production of circulating antibodies to Botulinum-A toxin in patients receiving repeated injection for dystonia [abstract]. Ann Neurol. 1988; 23:181.

\footnotetext{
Correspondencia:

Dr. Roberto Portillo Vallenas

Hospital Nacional Guillermo Almenara Irigoyen

Unidad de Neurofisiología

Av. Grau 800. Lima 13, Perú

Correo-e:drrobertoportillo@hotmail.com
} 